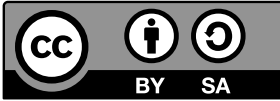

\title{
LE TOURBILLON DES SENTIMENTS AMOUREUX DANS LA POÉSIE DE MARCELINE DESBORDES-VALMORE OU LA POÉTISATION D'UNE HISTOIRE AMOUREUSE
}

Oubliées à tort pendant plusieurs siècles, les femmes de lettres, dont Christine de Pisan, Louise Labé, Madelaine et Catherine des Roches, Marceline Desbordes-Valmore et beaucoup d'autres, prêtaient à maintes reprises leur plume prolixe au service des épanchements du cœur. La voix féminine de la poésie courtoise, Christine de Pisan, s'est inscrite dans l'histoire comme la première Française à avoir jamais vécu de ses écrits. D’origine vénitienne, elle s'est permis, à la fin du XIV ${ }^{\mathrm{e}}$, début du $\mathrm{XV}^{\mathrm{e}}$ siècle, de célébrer le mariage et chanter, en son propre nom, l'amour pour son mari et le délice de la nuit de noces afin de montrer du doigt le concept erroné de l'amour courtois, vanté par les troubadours. Néanmoins, ce n'est qu'à la Renaissance que la scène littéraire assiste à « l'avènement » de la figure de la femme écrivaine/poétesse, incarnée par-dessus tout dans le personnage de Louise Labé, femme dotée de la liberté d'esprit, qui assumait pleinement sa féminité dans ses vers. À l'aube du XIX ${ }^{\mathrm{e}}$ siècle, le cercle s'agrandit avec Marceline Desbordes-Valmore, connue de nos jours comme prédécesseure de la poésie romantique.

Née le 20 juin 1786 à Douai dans une famille modeste et ruinée par la Révolution, elle n'a eu droit qu'à une formation rudimentaire. Marquée par la mort précoce de sa mère, elle s'est vu obligée de faire vivre sa famille. D'abord, elle a été engagée en tant qu'actrice et chanteuse, et ce n'est que bien plus tard, qu'elle s'est mise à écrire ses premiers poèmes. Aux environs de 1808, elle a rencontré un comédien, amateur de littérature, dont elle est tombée éperdument amoureuse. Si passionnée qu'ait pu être leur relation, les amoureux ont fini par se séparer. Unie par le mariage à Prosper Lanchantin, dit Valmore, en 1817, Marceline est entrée dans une époque de sa vie marquée par la pauvreté et de nombreux déplacements dus aux engagements professionnels de son mari, lui aussi acteur, mais malheureusement dépourvu de tout talent. Quatre enfants sont nés de ce mariage, dont seul Hippolyte survivra à Marceline, morte le 23 juillet 1859, à Paris, à la suite d'un long combat contre le cancer. 
L'hommage rendu par Marceline Desbordes-Valmore à son amant dans ses recueils poétiques a pendant de longues années intrigué les biographes et historiens littéraires qui avaient établi, séparément les uns des autres, une liste exhaustive de candidats possibles. De nos jours, le nom revenant le plus souvent est celui d'un certain Henri Latouche, ami de longue date de la famille Valmore.

Le premier recueil signé de sa main, Élégies, Marie et Romances, est sorti en 1819, annonçant ainsi une étoile montante de la poésie française. Classés en deux grands chapitres, les élégies l'emportent de beaucoup sur les romances en raison de la complexité de l'expression, du sentiment mélancolique et plaintif où Marceline excellait à merveille. Au cours de sa vie, elle a publié une dizaine de recueils, dont Élégies et Poésies Nouvelles (1824), Poésies en trois volumes (1830), Pleurs (1833), Pauvres Fleurs (1839), Bouquets et Prières (1843) et un dernier, posthume, Poésies Inédites (1860). La passion amoureuse, sa principale source d'inspiration se voit décortiquée avec une souplesse singulière ; Marceline pénètre avec la parole dans tous les coins de ce sentiment. En prosodie, elle était autodidacte, et toutes ces heures, passées auprès des textes qu'il a fallu mémoriser, ont fait également fleurir abondamment son imagination, ainsi que sa sensibilité romantique. Ses vers ne sont pas le travail d'un « poeta doctus ». Et pourtant, bien que simples, ils sont pleins de vivacité, de spontanéité, même de musicalité.

Après le décès de sa mère, Marceline s'est vue contrainte de retourner en France et d'entreprendre le métier d'actrice. Ainsi, en tant que jeune première occupée tous les soirs à mimer l'amour sur scène, elle a appris la prosodie et l'art de chanter. Bien qu'entrée dans le monde du théâtre en raison de son infortune, elle porte sur ce dernier un regard favorable ; à l'époque, c'était son refuge. Ayant souffert d'un mal mystérieux, Marceline, à l'âge de vingt ans, n'était plus en mesure d'interpréter des chansons. Ce handicap est très probablement apparu suite à la naissance de son fils Marie-Eugène. En vue de faire le deuil de cette perte, et en raison de son état de santé devenu déplorable, Marceline, suivant en cela les conseils de son médecin, s'est mise à écrire, à composer des vers. Elle s'en est exprimée dans une lettre adressée à Sainte-Beuve : "Je fus forcée de les (des idées) écrire pour me délivrer de ce frappement fiévreux, et l'on me dit que c'était une élégie. $\|^{1}$

Ayant connu Marceline personnellement et même failli épouser sa fille aînée, SainteBeuve cultivait une sincère admiration envers cette femme. D'après ses écrits, la société d'époque avait affaire à une poétesse tendre, instinctive et éplorée. Poétesse par amour, pour avoir aimé et pour avoir été aimée, sa disposition à composer des vers se trouvait indivisible de la passion. Après tant de pleurs, tant de gémissements de l'âme, ce talent qui, au fil du temps, s'en était abreuvé, ne fit que croître.

1 Sainte-Beuve, Portraits contemporains II, p. 106. 
Car je suis une faible femme,

Je n'ai su qu'aimer et souffrir ;

Ma pauvre lyre, c'est mon âme... ${ }^{2}$

Toujours souffrir ! Chanter toujours ! C'était cela son vrai rôle dans la création. Ainsi inspirée uniquement par sa propre expérience, Marceline se voyait étrangère à toute école d'art si bien que, tant de fois, ses vers font songer à de simples cris, plaintes et larmes. En écrivant, elle embellissait sa souffrance, elle voilait son chagrin de la mélodie la plus tendre. Peu de poètes connaissaient l'art de verser des larmes, voire des torrents de larmes; de pousser des cris déchirants ; d'exhaler des plaintes lamentables comme l'avait jadis maîtrisé Marceline. Dans « son genre », elle était une poétesse originale, chantant avec une voix poignante, pénétrante, avec une voix qui unissait en même temps la tristesse et l'ardeur, d'où notamment sa singularité. Elle est indiscutablement la poétesse méritant d'être classée parmi des grands noms de l'école romantique. "Pour comprendre toute la valeur du talent de Mme Valmore, il ne suffit pas d'avoir un goût pur, de se plaire aux belles expressions et aux belles images; il faut avoir l'instinct métaphysique de la poésie, savoir ce qu'elle est en soi, pénétrer jusqu'à son essence, $\|^{3}$ a noté Émile Montégut dans son article. Néanmoins, parvenir à une définition uniforme de ce que pourrait être la poésie à son origine ne me semble pour autant possible en aucun cas. Sauf si on l'explique par un cri, par des sons singuliers qu'on n'arrive plus à retenir, qui trouvent finalement leur chemin vers le monde extérieur par une agitation de l'âme, vu que c'est la passion la plus ardente qui fait vivre la poésie. Quand on lit ses poèmes, on constate que rares ou presque inexistants sont ceux ayant quelque chose de prémédité, tout nous est présenté à « son état cru », on a affaire à une émotion pure et première. Ses pleurs jaillissent sous l'emprise d'une émotion momentanée, raison pour laquelle ses propos sont assez souvent considérés comme dépourvus de toute cohérence.

Il se peut qu'un poème se trouve incarné dans le premier cri arraché par la douleur qu'afflige une personne adorée à l'excès, ailleurs, on le perçoit sous la forme de la passion et de d'une tendresse touchante, d'une supplication vis-à-vis du « malfaiteur ». À vrai dire, la lamentation qui devrait engendrer une élégie reste à son instance première, en un mot, elle n'est que l'élégie elle-même. Son art est un art purifié de tout artifice. Marceline ne s'exprime pas pour avoir un jour connu le chagrin amoureux, au contraire, son amour, sa souffrance, ils sont contemporains avec le moment de l'écriture. On assiste à un spectacle de la consternation, où elle nous communique la première impression de ressentis.

À ses yeux, la poésie produisait le même effet bénéfique, voire purificateur, que les larmes. Elle voyait dans la poésie une échappatoire lui permettant de donner libre cours aux sanglots et au déchirement qui l'étouffaient. Pleurer veut dire composer des vers. En

2 Marceline Desbordes-Valmore, Les Pleurs, 1833, pp. 203-204.

3 Emile Montégut, « Portraits poétiques : Mme Desbordes-Valmore », pp. 997-1016. 
fin de compte, sa poésie peut être qualifiée comme une œuvre de la nature. Ses poèmes, exempts de tout décor, nous laissent entrevoir une âme dénudée. Sans illustre héritage littéraire ou philosophique, la poétesse disposait d'une richesse particulière - intérieure : son amour, sa tendresse ainsi que ses douleurs.

Il y a des poétesses et poètes qu'il vaudrait mieux ne pas connaître dans leur intégralité du fait que toutes leurs œuvres ne sont pas forcément à la hauteur des grands auteurs. Ce n'est pas le cas de Marceline Desbordes-Valmore. Poétesse, elle l'est, grâce à sa sincérité, page après page. Comme déjà plusieurs fois souligné, son cœur et sa poésie n'existent jamais l'un sans l'autre, ("L'amour sera ma seule erreur ;/ Et pour la peindre en traits de flamme, / Je n'ai besoin que de mon cour ",) " "parce que la poésie se confond en elle avec la vie. ${ }^{5} \mathrm{C}^{\mathrm{e}}$ est pourquoi la tâche de citer une strophe ou bien une séquence de vers nous semble trop laborieuse ; chacune de ses pièces est unique et donc précieuse à sa manière. MOTIFS

La poésie lyrique se traduit en priorité par des expressions intimes. Cette « exaltation du Moi » enferme les notions dont l'amour, la nature, la solitude, la fuite du temps, on voit le sujet lyrique s'enfuir dans le monde onirique, il est abattu par la nostalgie pour un passé idéal, maintenant lointain et perdu. Dans ce sens-là, Marceline est la plus lyrique qu'il soit. Ce qui est singulier dans son œuvre poétique, c'est que son âme se trouve toujours seule ; jamais accompagnée par rien ni personne si ce n'est que par ses propres désillusions et chagrins. "Mais je suis seule, au moins, seule avec ma tristesse, / Et je trace, en rêvant, cette lettre pour toi... / Pour toi que j'espérais, que j'accuse, que j'aime! ${ }^{6}$ Aux Muses : «Livrez-lui vos trésors, ouvrez-lui votre temple, / A celui de l'Amour, seule, j’irai pleurer. »

Certes, dans sa poésie amoureuse, il apparaît bel et bien une autre personne, ses sœurs, un Tu ou un Lui - le menteur d'amour, l'ingrat, La Cause adorée de ses pleurs éternels. Pourtant ceux-ci ne sont jamais réellement présents. En plus, on a l'impression qu'il vient juste de partir. Aussitôt qu'il aura franchi le seuil de la demeure de Marceline, elle, plongée dans une solitude extrême, commencera, à son tour, sa « carmen miserabile ».

«Ma sœur, il est parti! Ma sœur, il m'abandonne! Je sais qu'il m'abandonne, et j'attends et je meurs ... ${ }^{7}$

\footnotetext{
4 Marceline Desbordes-Valmore, Élégies, Marie et Romances, p. 21.

5 Emile Montégut, « Portraits poétiques : Mme Desbordes-Valmore », pp. 997-1016.

6 Marceline Desbordes-Valmore, Élégies, Marie et Romances, p.18.

7 Poésies de Madame Desbordes-Valmore, tome I, 1830, p. 295.
} 
Ces monologues intérieurs d'une âme consternée ne consistent en effet que des cris et des plaintes ("Mais pourquoi, quand son amour cesse, I ne cesse-t-on pas de l'ai$\left.m e r »^{8}\right)$ : des éléments intrinsèques de la poésie lyrique pure. Il est clair qu'elle chantera toujours la même note poignante renvoyant de la passion malheureuse, pourtant cette passion ne manquera-t-elle jamais de lyrisme.

Dans les premiers recueils poétiques, sortis en $1819,{ }^{9} 1825^{10}$ et $1830,{ }^{11}$ avec presque un seul et unique thème qui est celui d'amour, tout est orage et tonnerre. La vie y foisonne. Néanmoins, les instants heureux ne sont que peu fréquents. Dans ses pièces, à de très rares exceptions, l'amour est perçu « sub speciae tragediae ». Bien qu'elle se sente profondément blessée par l'abandon de son bien-aimé, elle envisage ses propres douleur et chagrin avec une sorte d'allégrement. Elle a souffert le martyr et s'en est réjouie à point de s'y être abandonnée. La fureur amoureuse, cette fidèle compagne finit par la libérer, mais pas avant de lui avoir fatigué le cœur jusqu'au bout. Sous la griffe du regret, du chagrin et de l'incertitude, elle vit dans un état d'instabilité, la faisant vaciller entre l'espoir et désespoir, jusqu'à n'en plus distinguer l'un de l'autre. "Dans un trouble charmant je suivais l'Esperance ; / Elle enchantait pour moi les apprêts du concert ; Et je devais y pleurer ton absence! ".$^{12}$ "De ma jeunesse a flétri l'espérance : / Un orage a courbé le rameau délicat, / Et mes vingt ans passeront sans éclat. ${ }^{13}$ Elle préfère, et le crie à l'Amour, l'ignorance complète à une vérité, certes, désespérante, mais qui peut-être la ferait tourner la page. Puisque, pendant ces doux instants, il lui est permis d'espérer et jusqu'à ce que « cette lettre » sera restée intacte, Marceline sera aimée :" Mais, si tu dois détruire un espoir que j'adore, /Amour! de ce billet détourne ton flambeau; Par pitié, sur mes yeux attache ton bandeau, / Et laisse-moi douter quelques moments encore! " ${ }^{14}$

\subsection{Marceline et ses vues sur le bonheur personnel}

Force est de signaler également qu'elle ne s'abreuve que d'une simple passivité vis-à-vis du vécu. Marceline ne se bat pas, ne se défend pas, elle ne le maudit pas non plus - «Qu'il vive pour une autre et m'oublie à jamais. ${ }^{15}{ }^{5}$ 'où cette grandeur d'âme dont elle fait montre ? Même si ses prières ne sont jamais entendues et que leurs chemins ne se recroisent pas, au moins, qu'il retrouve le bonheur. Verbaliser la suite lui a coûté des larmes « Muses ! qu'il soit heureux, du moins par vos bienfaits! / Heureux sans moi... " ${ }^{16}$ Elle n'attend plus rien

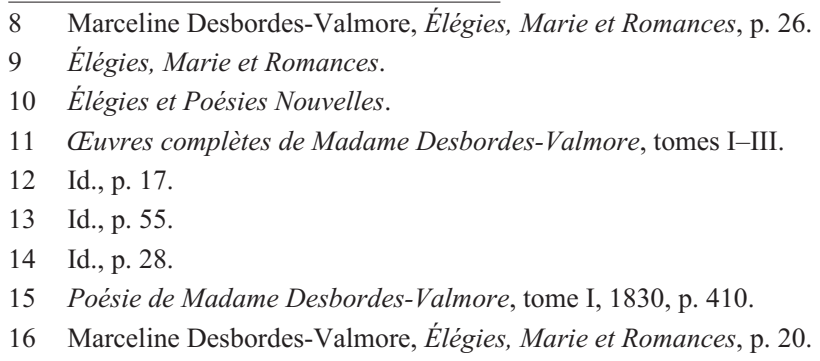


de l'avenir, si ce n'est de souffrir et chanter toujours en solitude, mais rassurée, sachant qu'il est heureux. «Si jamais, dans ma solitude, / Ton nom, pour toujours adoré, / Vient frapper mon coeur déchiré, / Qu'il adoucisse au moins ma tendre inquiétude ! / Que l'on me dise : il est heureux. / Oui, sois heureux, ou du moins plus paisible. ${ }^{17}$

Pour lui, au contraire, il y a encore de l'espoir, la vie lui promet de belles choses. Qu'il soit reconnu à sa juste valeur sans payer tout le mal qu'il lui a fait subir. "Le voilà donc fini mon court pèlerinage. / Ciel! que le sien plus beau soit ombrage de fleurs ;/ Et loin de le punir de mes tendres malheurs, / Qu'un suave laurier couronne son bel age... ${ }^{18}$ Incapable de garder de la rancune contre quelqu'un, elle accepterait de changer la place avec cet homme, puisque gravement malade, (si on la prend au sérieux), ou de subir toute punition pourvu que tout le mal lui soit épargné. «Et moi, je ne sais plus qui des deux fut coupable : / C'est moi mon Dieu! c'est moi si vous devez punir; oubliez le passé, je prends son avenir : / Dans la tombe qui s'ouvre, ah! laissez-moi l'attendre !. ${ }^{19}$ Sa parole ne manque pas de vraisemblance vu qu'elle lui apportait, sans le moindre doute, un amour fort et sincère; pour lui elle serait prête à donner la vie : "Qu'il vive de ma vie et je meurs sans regrets! / Ma vie hélas, c'est peu, mais il souffre et j'implore. / Jetez, jetez sur moi ce mal qui le dévore, / ... / Qu'il vive pour une autre et m'oublie à jamais. « ${ }^{20}$ Tout en elle n'est donc que résignation et souffrance ; elle s'effondre. Loin du dramatisme démesuré, sans le moindre reproche amer, elle fait face à l'aide des plaintes déchirantes. En chantant, elle implore la grâce, la sympathie et même le pardon auprès du tout puissant Amour du fait que son cœur endeuillé n'en peut plus. À ce moment-là, la douleur devient presque physique : "Pourquoi toute cette souffrance ne lui a pas été épargnée ? \$ Sans trêve, elle cherche des possibles explications à son calvaire; de sa part, aucun crime n'a été commis : « J'ai rencontré l'Amour, il a brisé ma lyre /... / Je t'ai chanté, lui dis-je, et ma voix faible encore, / Dans ses premiers accents parut juste et sonore ; / Pourquoi briser ma lyre? Elle essayait ta loi. / Pourquoi brûler mes vers? / Je les ai faits pour toi. $\rangle^{21}$ Ecrire des vers lui permet de se battre en vue d'atteindre ce bonheur tant désiré. Elle aspire à être aimée, mais pendant qu'elle nourrit ce désir suprême, elle refuse de croire qu'elle le sera un jour. Ses plaintes et alarmes, n'ayant apporté rien de bénéfique, n'engendrent que de l'inconstance et de la méconnaissance. Se voyant toujours malheureuse en amour, à travers ses écrits, elle constate : «Pour moi, il n'y a pas d'amour heureux! » Je l'ai prévu... j'ai voulu donc fuir : / L'Amour jamais n'eut de moi que de larmes. ${ }^{22}$

Marquée à vie par son calvaire amoureux, Marceline s'abstient d'un nouvel amour qui se serait sûrement mal fini. Cette expérience l'avait programmée à penser toujours le pire des affaires du cœur. «Inconstance ! affreux sentiment ! / Je t’implorais... je te déteste. /

17 Id., p. 60.

18 Marceline Desbordes-Valmore, Élégies et Poésies nouvelles, p., 47

19 Id., p. 50.

20 Id., p. 51.

21 Élégies, Marie et Romances, p. 37.

22 Id., p. 58. 
Si d'un nouvel amour tu me fais un tourment, / N'est-ce pas ajouter au tourment qui me reste ? /... / Je tremble d'être heureuse et je verse des larmes. " ${ }^{23}$ Paradoxalement, quoique les êtres humains ne puissent généralement qu'essayer, la première déception amoureuse a été une fois de trop pour la poétesse hypersensible. "Oui, de trop de regrets, l'espérance est suivie : / Je renonce au bonheur, j'ai perdu mes beaux jours. / Adieu, le charme de ma vie! ${ }^{24}$ De rares instants heureux ("Un bienfait de l'Amour a changé mon destin : Oh! qu'il m'a révélé de touchantes nouvelles ! Il m'aime, il m'aime encore ! ... »²5), si elle arrive à y faire allusion, ils ne dépassent jamais le cadre bien figé, conditionné par la privation d'amour. En effet, tout n'était qu'un songe. Elle ne dit pas : » J'étais heureuse, » mais : "Je rêvais de bonheur. " ("Ivre de toi, je rêvais le bonheur, / Je rêvais! ... tu m'as éveillée! / Que ce réveil va me coûter de pleurs! $\gg{ }^{26}$ Ce qui nous touche d'avantage, en tant que lectrices et lecteurs, c'est le moment du réveil, lorsque Marceline parvient à y mettre un nom : elle était la seule à avoir envisager un futur commun et à avoir aimé ou bien elle était la seule à aimer encore. «Que faisait-tu, mon idole chérie, / Quand ton absence éternisait le jour? / Quand je donnais tout mon être à l'amour, / M'as-tu donné ta rêverie? $"{ }^{27}$ Lui, à son tour, poursuivra le cours de sa vie, il retrouvera le bonheur en multipliant les rencontres amoureuses. "Votre bonheur, hélas! sera d'être volage, / Vous séduirez encor dès qu'on vous entendra ; / Vous ferez le tourment de qui vous aimera. " ${ }^{28}$ Pourtant, rien ni personne ne pourrait empêcher Marceline d'accomplir les vœux, faits à son ami - Nul homme ne la fera vibrer comme il l'avait fait, car elle était née pour n'aimer qu'une fois. "J'ai repris le serment d'être à vous pour toujours ; / Mais mon âme un instant fut unie à la vôtre, / Et je le sens, jamais un autre / N'aura mes vœux, ne fera mes beaux jours. " ${ }^{29}$ Et mon cœur fut créé pour n'aimer qu'une fois !» ${ }^{30}$

\subsection{Marceline sous l'emprise de son amant}

Au premier regard, aux premiers mots échangés, Marceline a succombé sous le charme de cet homme. Ce n'était pas une rencontre comme les autres, mais plutôt une rencontre à part, subite et violente. Comme tous les grands romantiques, elle a été victime d'un puissant coup de foudre qu'elle a fini par transposer en vers avec une sensibilité distinguée. Force est de constater que son élu avait un caractère plutôt difficile, pour ne pas dire insupportable. Il paraît qu'à un moment donné, il a tâché de transformer leur vie de couple en un enfer terrestre,

\begin{tabular}{ll}
\hline 23 & Id., p. 48. \\
24 & Id., p. 62. \\
25 & Id., p. 30. \\
26 & Id., p. 21. \\
27 & Id., p. 21. \\
28 & Id., p. 26. \\
29 & Id., p. 25. \\
30 & Id., p. 50.
\end{tabular}


suggérant vivement à Marceline de ne plus l'aimer, vu que lui n'en était pas capable à cause de sa maladie noire qui reléguait le bonheur à l'état de rêve lointain. Blessée par ses outrances, Marceline blâme l'Amour de l'avoir enchaînée à tout jamais à son malfaiteur. Se voyant dépourvue de force, elle n'est pas en mesure de le quitter. «De te quitter donne-moi le courage. ${ }^{31}$ Durant les rares moments de lucidité, elle en profite pour nous communiquer sa prise de conscience : elle s'est rendu compte de l'effet néfaste de cet amour et envisage la séparation en tant que la seule et unique issue possible : "Laisse-moi respirer du trouble qui m'accable ; / Laisse-moi retrouver mon cœur ! / Séparons-nous, je suis trop attendrie : Sur ce cour agité ne pose plus ta main. Va... ${ }^{32}$ Cependant, au moment même qu'elle y songe, une sorte de servilité reprend le contrôle; habituée à cet esclavage, qui la suit comme une ombre, comment son âme tiendra-t-elle? «Que sert de lui donner ma vie, / S’il est heureux sans moi ? / Que deviendra l'amour dans mon âme asservie... " ${ }^{33}$ "Qu'il m'était cher ! que je l'aimais ! / Que par un doux empire il m'avait asservie ! " ${ }^{34}$

Dans le but de ne jamais manquer de son affection au quotidien, chaque pas, chaque souffle, tout était conditionné par lui. C'était son tyran, son maître, son inspiration, sa raison de vivre. «Ces jours consacrés à vous plaire, / Ces vœux si tendres et si doux, / Et toujours inspirés par vous, / Désormais qu'en pourrai-je faire? " ${ }^{35}$ Progressivement, toute cette dépendance affective - car, effectivement, elle n'aimait que « par lui » - dont elle souffrait a débouché sur le déni total de sa propre personne. «Quand ta bouche m'apprit, je ne sais quel serment : / Qu'importe les serments ! Je n'étais plus moi-même, / J'étais toi. J'écoutais, j’imitais ce que j'aime: / Mes lèvres, loin de toi, retenaient tes accents, / Et ta voix dans ma voix troublant encor mes sens. $\|^{36}$ La fusion, au moins de la part de Marceline, semblait être complète, à tel point qu'elle n'appartenait plus à la sphère terrestre : "Qu'ai-je dit ? Notre amour, c'est le ciel sur terre. " ${ }^{37}$ Avec lui, Marceline ressentait un vrai bonheur qui donnait les justes couleurs à ses jours, dont rien ni personne ne pouvait ravir l'harmonie. Autrement dit, ni son tempérament, ni le fait qu'il l'ait cachée à sa famille, parce qu'elle exerçait un métier peu flatteur, ni le fait qu'il ait refusé de reconnaître leur enfant, ne pouvait la détourner de lui.

Tous les doutes qu'on aurait pu garder viennent de se dissiper. Effectivement, à ce moment-là, leurs cœurs ne battaient plus à l'unisson, même si, au départ, les sentiments avaient été réciproques. Il n'a jamais envisagé un futur commun : "Jouant avec mon cour qu'il déchirait... hélas, / Il parlait de bonheur sans parler de tendresse. / Il parlait d'avenir, et ne me nommait pas! « ${ }^{38}$

\footnotetext{
31 Élégies, Marie et Romances, p. 13.

32 Id., p. 14.

33 Élégies, Marie et Romances, p. 20.

34 Élégies et Poésies nouvelles, p. 48.

35 Élégies, Marie et Romances, p. 26.

36 Élégies et Poésies nouvelles, p. 9.

37 Id., p. 10.

38 Id., p. 47
} 


\subsection{Son départ}

Leur histoire a connu deux séparations, néanmoins certains assure qu'il y eut une deuxième réconciliation.

C'est par orgueil qu'aucun d'entre eux n'a voulu faire son mea culpa et renouer ainsi la relation en premier. De ce fait, la première rupture semblait déjà définitive ( « semblait », car, quelques années plus tard, leurs chemins allaient se recroiser ; au total, ce douloureux bonheur n'aura duré qu'un an et demi, deux ans au maximum.) À peine avaient-ils rompu que son bien-aimé s'en allait en Italie et, pendant ce temps, Marceline, pleurant à chaudes larmes, s'est réfugiée à Rouen. Bien que certains prétendent que les amoureux avaient continué à échanger même après cette rupture (elle aurait reçu une lettre d'Italie), on n'en a pas, à part de ce qu'elle nous fait connaitre à travers ses poèmes, la moindre preuve, d'autant plus que Marceline nous dit dans « le Retour chez Délie»: "Ne me reprochez plus ma fuite et mon silence. $"{ }^{39}$ Faut-il croire alors que Marceline s'était promis à un silence absolu ? En tout état de cause, Marceline se voyait de retour à Paris pour reprendre son métier d'actrice, et malheureusement, elle y est tombée dans les bras de son amant. Même s'il paraît que, tout au début, Marceline essayait de garder ses distances, le fait de fuir et de renoncer à son amoureux aurait exigé d'elle une force surhumaine. Une fois de plus, elle rêvait de l'impossible. Apparemment, les amants s'étaient promis de ne jamais s'adresser la parole, de s'oublier complètement. Une deuxième fois, elle prononce le nom du grand coupable, de celui qui lui a « coûté tant de larmes » : l'orgueil.

La séparation, l'abandon, mais surtout la crainte extrême de l'abandon ; la hantise qu'il ne revienne jamais plus, une fois que les portes se seront refermées derrière lui, marque dès le début un grand nombre de poèmes. Quoi que «le méchant» dans l'histoire soit le soir séparant les jeunes amoureux : "En te disant adieu chaque soir je soupire ; I Ah! puissions-nous bientôt désapprendre à le dire! / Ce mot, ce triste mot n'est pas fait pour l'Amour, $»^{40}$ ou le mauvais temps - un orage prophétique du futur malheur : "Quels éclairs ! quel bruit sourd! ... ne t'en vas pas, j'ai peur! Les cris aigus de l'hirondelle / Annoncent le danger qui règne autour de nous /... Reste, mon bien-aimé, reste, je t'en conjure ; /.. / Tu t'éloignes... tu crains un danger que j'ignore ? $\|^{41}$ la menace du futur départ est omniprésent. Désespérée, elle s'adresse même aux muses, en les implorant de lui rendre son amour : "Votre empire a troublé mon bonheur le plus doux. / Muses! rendez-moi ce que j'aime ! /... Ce n'est plus pour moi qu'il délirel Il a banni mon nom de ses écrits touchants. $»^{42}$

Dans plusieurs poèmes, on a l'impression que Marceline revit le moment douloureux de la séparation; qu'elle se force à y penser afin de recréer les derniers instants passés dans sa compagnie, d'entendre ses derniers mots. Néanmoins le mot ne sort jamais de sa

39 Poésies de Madame Desbordes-Valmore, tome II, 1830, pp. 265-266

40 Id., p. 13

41 Id., p. 14

42 Id., p. 19 
bouche. Lui dire adieu l'aurait entraînée dans une défaillance complète. "Point d'adieu, non! ce mot est l'effroi d'un cœur tendre. $»^{43}$

"Pale, presque à genoux, suppliante, craintive, / J'ai dit... Je n'ai rien dit, mais on entend les pleurs. / Et ce morne silence ou parlaient les douleurs, / Ce cris, prêt d'entrouvrir le sein qui le captive. $»^{44} \mathrm{~A}$ force d'avoir souffert si longtemps, elle n'arrive plus à extérioriser ses ressentis. Seules les douleurs, accompagnées de larmes, osaient briser ce silence glaçant, régnant lors de cette «fraction». "C'en était fait, ma sœur. De mes larmes suivie, / Je repris la raison sans reprendre la vie. / J'écoutai... de ses pas le bruit s'était perdu, / J'étais seule... ${ }^{45} \mathrm{Ne}$ faisant que du surplace, elle se voyait obligée, coûte que coûte, de poursuivre cette existence qui ne rimait à rien. Bien qu'endeuillée, Marceline n'avait pas de choix, ni de temps, pour essuyer ses larmes, d'autant plus qu'il fallait faire vivre sa famille et d'avantage son père. Cependant, reprendre la vie avec tout ce qu'elle promet ne lui était plus accessible. Partant de sa logique, où le lien entre aimer / (d'être aimée) et de vivre repose sur la synonymie, l'on arrive à la conclusion suivante : sans amour (sans Lui), elle s'identifie à « une femme morte en vie », car seul l'amour pourrait la garder en vie. La nouvelle réalité s'affiche du jour au lendemain : «Il ne viendra pas! », et elle l'endure, car elle n'a pas de choix. «Toi tu ne viens jamais! Qu’importe que je meure. / Les minutes en vain volent autour de l'heure; / Et l'heure, en les comptant, fait tomber sans retour / Les mois, les ans, la vie! et sans toi, sans amour. $»^{46}$ Dorénavant, les scènes où Marceline se peint morte vont défiler crescendo.

\subsection{Après son départ, Marceline tombe dans un état second}

Après son départ, une fois que toute trace de lui s'est perdue, lorsque le regain d'espoir n'était plus envisageable, la conscience de Marceline a commencé à lui imposer des images délirantes. On y voit une Marceline, empoissonnée par ses rêves fiévreux, la faisant croire qu'il s'approche. "Dans le demi-sommeil où je tombe rêveuse, /Je te crains, je t'espère et je te sens venir ;/ Tu parles, mais si bas! une oreille amoureuse / Peut seule entendre et retenir : "Veux-tu, mais ne dis pas que l'heure est trop rapide, / Veux-tu voir la montagne et le courant limpide? / Veux-tu venir au pied du grand chêne abattu? " / Moi je ne réponds pas pour écouter : "Veux-tu? " / Veux-tu voir notre image au bord des flots penchée? / Ne tremble pas tout dort ; l'écho même s'est tu. / Et mon refus se meurt en écoutant : "Veux$t u . ~{ }^{47}$ Autrefois incarnation de l'Amour, paré d'une voix tendre, maintenant il se voit réduit à un simple écho, faible et craintif s'affichant devant elle. Le cruel ne la laisse pas faire son deuil en paix, il la perturbe en faisant jaillir quelques vestiges de l'ancien amour. Tout pour

\footnotetext{
43 Élégies et Poésies nouvelles, p. 56

44 Id., p. 47

45 Id.

46 Élégies et Poésies nouvelles, p. 15

47 Élégies et Poésies nouvelles, p. 5
} 
affirmer son éternelle emprise sur elle : elle ne l'oubliera pas. «Pourquoi ce faible écho, craintif comme nos vœux, / Dit-il contre mon cœur : "Bonsoir, ma bien- aimée? " / Ah! je t'en prie, il ne faut plus venir / Redemander mon âme presque heureuse : / Je crains de toi jusqu'au ton souvenir ; / Loin du danger je suis encor peureuse ... " ${ }^{48}$ Avant assurant son rôle du maître de sa vie, maintenant comme une entité protéiforme, il accapare son sommeil, en devenant une ombre, un fantôme - celui qui fait peur ! Bien sûr, il est impossible qu'elle l'échappe. "Mais à travers mes pleurs et cette clarté sombre, / J'ai vu paraitre une ombre, / Autrefois mon idole, aujourd'hui mon effroi : / Cette ombre était la sienne, elle avançait vers moi. " ${ }^{49}$ Dans son délire, Marceline s'imagine des rencontres fictives, elle revêt cette ombre de chair humaine, parce que c'est inconcevable pour elle de continuer à vivre sans lui. "L'ombre alors me repousse et m'entraine a-la-fois. / Oubliant ma faiblesse et ma fièvre brûlante, /Partout pour la saisir, j'étends ma main troublante .../ J'ai couru, j'ai suivi des sentiers que j’ignore ;/ Demi-nue, insensible au souffle de l'hiver, / J'obéissais, mourante, à ce guide si cher : Il ne m'appelait plus, j'obéissais encore. / La pluie a longs torrents inondait le chemin:/ Le vent soufflait : " Demain! n'attends pas à demain! " / Et je tombe à sa porte, et presque évanouie, / Par l'éclat des flambeaux je m'arrête éblouie. /... C'était... l'ai-je rêvé ! " ${ }^{50}$ Dans son rêve, elle s'efforce en vain d'attraper cette ombre. Ces longues attentes stériles se transforment en ombres qu'elle chasse ardemment et qui, en retour, la persécutent jusqu'à la faire tomber dans le délire. Ainsi finit-elle par le voir partout et à toute heure, "Le soir, à l'horizon, ou s'égare ma vue, /Tu m'apparais encore, et j'attends malgré moi:/ La nuit tombe... ce n'est plus toi ; Non! C'est le songe qui me tue. $\|^{51}$

\subsection{Haïr, mais comment ?...}

La voir plongée dans un monde qui n'est nullement le sien, où règnent colère, injures et haine, risque de nous faire détourner le regard et de la taxer d' "impudique ». Bien que toujours affligée, ce n'est pas dans sa nature de s'en prendre à l'Amour, ni de courir après la gloire. Malgré une prise de distance, derrière cette troisième personne se cache bel et bien Marceline. «... Punis ton injuste maitresse : / Elle a maudit l'amour ; j'en suis tremblante encor. / Elle a maudit ses pleurs, ses tourments, son ivresse, / Et sa révolte a pris l'essor. "/.../ L'amour seul est rapide, ingrat, sans souvenir ; / Il devance, il dévore, il détruit l'avenir : / Je déteste l'amour. / Je veux aimer la gloire ;/ Elle promet des biens : / Je tâcherai d'y croire. ${ }^{52}$ D'habitude elle chérit, elle est aimante avec ses maux et ses pleurs. De plus, en composant des vers, elle en vient parfois à leur rendre

\begin{tabular}{ll}
\hline 48 & Id. p. 6 \\
49 & Id., p. 23 \\
50 & Id., p. 25 \\
51 & Id. p. 10 \\
52 & Id., p. 13
\end{tabular}


hommage : "Oui je sens que mes pleurs avaient pour moi des charmes, / Et que mes maux étaient mes biens! $\|^{53}$ Elle dit à Délie, à son de confiance, : "N'enviez plus à votre amie / Un plaisir aussi douloureux : / Ravir la plainte aux malheureux, /C'est leur dire : Quittez la vie, " ${ }^{54}$ ou dans un autre poème : "Contre tous les plaisirs d'une nouvelle flemme / Je n'échangerais pas mes pleurs. ${ }^{55}$ Peut-être la haine n'était-elle qu'une des étapes à franchir sur ce long chemin de guérison. Car, in fine, et elle l'écrit noir sur blanc : elle n'est pas capable de hair. " / Je hais tout! / Mais bientôt elle n'eut plus de voix, / Que pour former ton nom, pour t'appeler cent fois ;/ Elle cherchait en vain sa colère exhalée:/.../ En vain l'écho trompé disait : Je veux haïr ».56

\subsection{Marceline se voit morte ...}

Se voyant défaillante, sans aucun espoir d'occuper un jour à côté de lui une autre place que celle de maîtresse : "Blessée au cour d'un trait dont je ne puis guérir, / Sans prétendre aux doux noms et de mère et d'épouse, / Il me faut donc mourir ! $\left.{ }^{57}\right)$, son dernier souffle, Marceline en use à seule fin de pousser ses derniers cris - ses dernières élégies d'un cœur blessé à mort : "Cruel! en me quittant, tu me verrais mourir. $\|^{58}$ Lorsque son chagrin eut gagné une ampleur importante, ce mal-être immense s'est répercuté le long de tout son organisme. Même les larmes avaient cessé de couler : c'était une fois de trop. «Mes pleurs se desséchaient à leur source brulante, /Mon sang ne coulait plus, j'étais pâle, mourante ; / Mes yeux désenchantés repoussaient l'avenir... $\|^{59}$ "C'est la mort que je veux avant elle. / Tout est détruit : lui-même il n'est plus le bonheur. / Il brisa son image en déchirant mon cour. ... il n'a pas vu ma main faible et glacée, / Rassembler mes cheveux pour voiler mon affront: / Il n'a pas vu la mort par lui-même tracée... I Aveugle! il n'a pas vu se troubler et s'éteindre, / Mon cil long-temps fermé. ${ }^{60}$

Comme la goutte qui fait déborder le vase, la larme en trop possède la force de creuser un abîme encore plus profond. Si elle seule éprouvait un malheur inexplicable, c'est que, par analogie, elle était également la seule dans son couple à avoir aimé. C'est pourquoi elle ne parvient pas à intellectualiser cet acte dépassant l'entendement et qui se traduit chez elle par les images les plus morbides, dont celle où l'on la voit dans sa tombe - la nouvelle qu'elle venait d'apprendre lui a coûté la vie - ou même, comme dans les vers suivants, où Marceline se plaint de contempler sa tombe ignorée : "Au bord de ma tombe ignorée, /

\footnotetext{
53 Élégies, Marie et Romances, p. 48

54 Id., p. 54

55 Id., p. 61

56 Élégies et Poésies nouvelles, p. 13

57 Élégies, Marie et Romances, p. 56

58 Id., p. 14

59 Id., p. 50

60 Id.
} 
Ciel! par cette langue acérée, /Faut-il qu'un nom trop cher puisse m'atteindre encor/Pour m'apprendre (nouvelle affreuse!) / Que j'étais seule malheureuse, / Et qu'on m'oublie avant ma mort ! / Du plus sincère amour quel châtiment terrible! " ${ }^{61}$

Néanmoins, son imagination se nourrit davantage de ses plaies non cicatrisées ; elle ne trouvera la paix qu'une fois morte, car uniquement la mort pourra éteindre sur sa cendre l'amour maladif dont elle se trouvait prisonnière. " Mais je compte, j'attends que moins inexorable / Une heure, la dernière à mes maux secourable, / Éteigne sur ma cendre un importun flambeau, / Et défendre à l'amour de troubler mon tombeau. $»^{62}$ Ce qui est peutêtre même plus frappant, c'est que Marceline se lamente de ne pas être morte avant, lorsque l'Ingrat l'aimait encore. Ainsi, la mort lui aurait été douce, sachant que les larmes de son bien-aimé auraient arrosé les fleurs poussant sur sa tombe. "Qu'il m’eut été doux de mourir / Pour l'amant dont les pleurs me suppliaient de vivre. ${ }^{63}$ "Ne meurs pas, disait-il, ou je meurs avec toi ! » / ... / Sous ses pleurs j'allais m'éteindre heureuse, / J'allais mourir aimée. ${ }^{64}$ Pourquoi insiste-t-elle tant sur ce désir presque pervers de se voir morte ? Parce qu'elle s'est emmurée dans sa tête l'idée qu'après l'avoir vue morte, le Cruel se serait repenti. Ses yeux sont voilés de pleurs de bonheur, de pleurs d'amour, car la mort lui a rendu l'affection de son ami.

\section{4}

\section{CONCLUSION}

L'orage s'apaise, mais elle en sort gravement touchée. Marceline ne se remet pas et ne se remettra jamais, s'il s'avère que ses écrits sont fiables. Des recueils, tels que Les Pleurs et Pauvres fleurs n'en sont qu'une preuve probante où ce tendre cœur de Marceline, ensevelie par ses pleurs, prend la parole. Sa « carmen miserabile» se transforme ici en une expression de la plus poignante lassitude; quoique toujours en vie, Marceline semble être détachée de sa propre personne. Elle est « morte » tout en poursuivant sa vie.

Marceline Desbordes-Valmore appartenait à une classe d'individus à part, sensibles et meurtris dès le jour de leur naissance. L'infortune et le malheur l'ont élevée à leur exemple de sorte que le bonheur paraît être une abstraction, malgré son acharnement à l'atteindre dans l'écriture comme dans la vie réelle. Ses vers, si monotones qu'ils puissent sonner aux oreilles de certains, restent bien gravés dans la mémoire en raison de leur note languissante, sortant directement du cœur. Derrière ses œuvres se cache une poétesse qui nous offre un spectacle en vers à son état primaire. Par manque de fard et d'une certaine habileté, ses vers, écrits dans un style décharné, nous offrent une poésie sincère et originale.

\footnotetext{
61 Id., pp. 16-17

62 Id., p. 37

63 Id.

64 Id., p. 38
} 


\section{BIBLIOGRAPHIE}

AMBRIERE, Francis (1987) Le siècle des Valmore : Marceline Desbordes-Valmore et les siens, II. 1840- 1892. Paris : Éditions du Seuil.

BOULENGER, Jacques (1909) Marceline Desbordes-Valmore D’après ses papiers inédits. Paris : Arthème Fayard, éditeur.

DESBORDES-VALMORE, Marceline (1819) Élégies, Marie et Romances. Paris : Imprimerie de Richomme.

DESBORDES-VALMORE, Marceline (1825) Élégies et Poésies Nouvelles. Paris : Imprimerie de Firmin Didot.

DESBORDES-VALMORE, Marceline (1830) Poésies de Madame Desbordes-Valmore, tome I-III. Paris : A. Boulland, Libraire-Editeur.

DESBORDES-VALMORE, Marceline, (1860) Poésies Inédites de Madame Desbordes-Valmore, par M. Gustave Revilliod, Genève : Imprimerie de Jules Fick.

DESBORDES-VALMORE, Marceline (1820) Poèmes de Mme Desbordes-Valmore. Paris : François Louis, Libraire.

DESBORDES-VALMORE, Marceline (1833) Euvres de Madame Desbordes Valmore. Bruxelles : E. Laurent, imprimeur-éditeur.

DESBORDES-VALMORE, Marceline (1833) Les Pleurs, poésies nouvelles. Paris : Charpentier, Libraire.

DESCAVES, Lucien (1910) La vie douloureuse de Marceline Desbordes-Valmore. Paris : Éditons d'art et de littérature.

GIRAUD, Victor (1935) « Revue littéraire : Marceline », Revue des Deux Mondes vol. 29, no. 1, pp. 218-230. <https://www.jstor.org/stable/44848411> (consulté le 15 juillet 2020)

LEMAITRE, Jules (1899) Les contemporains : Etudes et Portraits littéraire, septième série. Paris : Société française d'imprimerie et de librairie. pp. 1-46.

MONTEGUT, Emile (1860) « Portraits poétiques : Mme Desbordes-Valmore », Revue des Deux Mondes, Vol. 30, n. 4. pp. 997-1016. <https://www.jstor.org/stable/44728016 $>$ (consulté le 20 avril 2019)

POTEZ, Henri (1897) « La poésie de Marceline Desbordes-Valmore », Revue d'Histoire littéraire de la France, ${ }^{\circ}$ 4, pp. 481-523. <https://www.jstor.org/stable/40517691> (consulté le 20 juillet 2020)

RIVIERE, Benjamin (1896) Correspondance intime de Marceline Desbordes-Valmore, tome I. Paris : Alphonse Lemerre, Editeur. pp. 134-135.

ROGER-VASSELIN, Bruno (2004) « Louise Labé et l'écriture au féminin », L'information littéraire, Vol. 56, $\mathrm{n}^{\circ} .4$, pp. 8-17. <https://www.cairn-int.info/revue-1-information-litteraire-2004-4-page-8.htm> (consulté le 3 novembre 2020)

SAINTE-BEUVE (1889) Portraits contemporains par C.-A. Sainte-Beuve. Paris : Calmann Lévy, Editeur. pp. 91-114. 
SAINTE-BEUVE (1833) « Poètes et romancières modernes de la France. VII. Mme Desbordes-Valmore », Revue des Deux Mondes, DEUXIÈME SÉRIE, 3, n 3. pp. 241-255. <https://www.jstor.org/stable/44688351> (cnsulté le 20 juillet 2020)

\section{POVZETEK}

\section{VIHAR LJUBEZENSKIH OBČUTIJ V POEZIJI MARCELINE DESBORDES-VALMO- RE ALI POETIZIRANA LJUBEZENSKA ZGODBA}

V pričujočem prispevku bomo posvetili pozornost zgodnji ljubezenski poeziji Marceline Desbordes-Valmore, kakor tudi njeni osebni zgodbi, zaznamovani z viharno zvezo, ki žal ni dočakala srečnega epiloga. S pomočjo izbranih verzov bomo osvetlili »dar joka«, ki ji ga je s svojim odhodom poklonil ljubimec, čigar identiteta navkljub različnim predvidevanjem ostaja skrivnost. Sainte-Beuve, ki je Marceline osebno poznal, jo je opisal kot zelo nežno, instinktivno in čustveno pesnico, ki je pisala zaradi ljubezni; zato ker je ljubila in ker je bila nekoč ljubljena ter nenazadnje, ker je vse to izgubila. Marceline se je znotraj »romantične šole« uveljavila s preprostostjo in iskrenostjo izraza, s poezijo, kipečo od čustvenega naboja; uspelo se ji je približati esenci lirske poezije. Četudi bi ji želeli pripisati patetičnost in solzavost, ji izumetničenosti nikakor ne moremo očitati. Pri motivni analizi se bomo opirali na epizode iz njenega življenja, obenem pa bomo pod drobnogled vzeli ljubezensko čustvo, primaren navdih v začetnih letih pesnikovanja. Sledili bomo odmevom, ki so jih v verzih pustili nerazumevanje, lažen up, razočaranje, neutolažljivost, žalost, strah ob misli na poslednje slovo, itd. ... Spomnili se bomo delirijev, sanj in prividov, v katerih ga ponovno vidi ter sliši, a ob tem se prizadejana bolečina le še poglobi. Nazadnje bomo omenili postopno spreminjanje razpoloženja, ki zavlada $\mathrm{v}$ pesmih, postaja zmeraj bolj temačno in morbidno, s tem pa naznanja tematski prelom v nadaljnjih zbirkah.

Ključne besede: Marceline Desbordes-Valmore, pesnica, dar joka, ljubezenska poezija, romantika

\section{ABSTRACT}

\section{A WHIRLWIND OF EMOTIONS IN THE POETRY OF MARCELINE DESBORDES- VALMORE OR THE POETISATION OF A LOVE STORY}

The subject of this paper is not only the love poetry of Marceline Desbordes-Valmore, which belongs to her early creative phase, but also her personal story, which was marked by a stormy relationship that unfortunately did not come to a happy end. Sainte-Beuve, who knew Marceline personally, described her as a very gentle, instinctive, and emotional poet. Through selected verses we examine her gift of "weeping", which she received with the departure of her lover, whose 
identity remains a mystery despite much speculation. Marceline established herself in the "Romantic school" with her simplicity and sincerity of expression, with poetry that boils with emotion. She even managed to approach the essence of lyrical poetry. While we can attribute pathos and sadness to her work, but there is no trace of artificiality in these verses. When analysing the motifs, we draw on episodes from her life, and at the same time look at her most important inspiration in the first years of her work, the power of love. We follow the effects that misunderstandings, confusion, disappointment, desolation, sadness etc. have left in the verses. We consider the deliriums, dreams, and illusions in which she sees and hears her lover again, but without causing her physical exhaustion. Finally, our discussion touches on the gradual change of mood that prevails in the poems. They become dark and morbid, and herald a thematic break that occurs in her following collections.

Keywords: Marceline Desbordes-Valmore, poet, love poetry, biographical reading, Romanticism 